\title{
Calculation of the Electronic Properties of Neutral and Ionized Divalent-Metal Clusters
}

\author{
M. E. Garcia, ${ }^{1}$ G. M. Pastor ${ }^{2}$ and K. H. Bennemann ${ }^{1}$ \\ ${ }^{1}$ Institut für Theoretische Physik der Freien Universität Berlin, Arnimallee 14, W-1000 Berlin 33, FRG. \\ ${ }^{2}$ Institut für Theoretische Physik der Universität zu Köln, Zülpicher Str. 77, W-5000 Köln 41, FRG.
}

Received 15 September 1992

\begin{abstract}
Abstraci. The electronic properties of neutral and ionized divalent-metal clusters have been studied using a microscopic theory, which takes into account the interplay between van der Waals (vdW) and covalent bonding in the neutral clusters, and the competition between hole delocalization and polarization energy in the ionized clusters. By calculating the ground-state energies of neutral and ionized $\mathrm{Hg}_{n}$ clusters, we determine the size dependence of the bond character and the ionization potential $I_{p}(n)$. For neutral $\mathrm{Hg}_{n}$ clusters we obtain a transition from van der Waals to covalent behaviour at the critical size $n_{c} \sim 10-20$ atoms. Results for $I_{p}\left(\mathrm{Hg}_{n}\right)$ with $n \leq 20$ are in good agreement with experiments, and suggest that small $\mathrm{Hg}_{n}^{+}$clusters can be viewed as consisting of a positive trimer core $\mathrm{Hg}_{3}^{+}$surrounded by $n-3$ polarized neutral atoms.
\end{abstract}

PACS: $36.40+d, 71.30+h, 33.80+E h$

In the last years much attention has been focussed on the study of divalent metal clusters[1-8]. These clusters exhibit a particularly rich size dependent electronic structure. This begins with the closed-shell $s^{2} p^{0}$ electronic configuration of the atom, changes dramatically for increasing cluster size $n$, and finally converges to the bulk behaviour, where the s-and p-bands overlap, giving rise to the known metallic properties of the solid[4-6]. In particular, for neutral $\mathrm{Hg}_{n}$ clusters, the existence of a transition from van der Waals (vdW) to covalent to metallic bonding for increasing cluster size is supported by experiments[1-3] and theoretical calculations[4-6].

First, to discuss the physics of the problem, let us recall the main attractive interactions between neutral divalentmetal atoms. The vdW interactions are the interactions between mutually induced dipoles involve mainly intra-atomic $s p$-excitations[9]. This means that the valence electrons remain localized aronnd the atomic cores. In contrast, the covalent bonding arises from interatomic sp hopping, which involves interatomic electronic charge fluctuations. Note that the importance of covalency (i.e., the covalent bond-order $\left.\left(c_{i, \sigma}^{+} c_{j p o}\right)\right)$ is regulated by the strength of the Coulomb interactions between electrons, since interatomic charge fluc- tuations increase the Coulomb repulsion energy. The fact that the same valence electrons take part in both vdW- and covalent-bonding mechanisms gives rise to the interplay between these two microscopically different types of binding.

Several experiments have been performed in order to detect this size dependent change of the bond character in $\mathrm{Hg}_{n}[1-3]$. Among them, the results for the size dependence of the ionization potential[2,3] are particularly challenging for theory, since here not only the electronic structure of the neutral cluster but also that of the cation is involved.

In a recent paper we have developed a theory to describe the change of the bond character in divalent-metal clusters[5]. There we have shown that the interplay between the $\mathrm{vdW}$ in teractions and the itinerancy of the valence electrons depends on cluster size $n$ and can give rise to a transition from $\mathrm{vdW}$ to covalent bonding for increasing $\boldsymbol{n}$ and, moreover, that such a transition occurs for $\mathrm{Hg}_{n}$ clusters. In this paper results are presented which demonstrate that our theory also accounts for the size dependence of the electronic properties of ionized divalent-metal clusters.

The Hamiltonian which describes the main dynamics of the valence electrons in divalent-metal clusters can be written as $[5,6]$

$H=H_{v d w}+H_{c o v}+H_{Q-P}+H_{P-P}$.

The operator $H_{v d w}$ refers to the vdW interactions and the covalent interactions are described by $H_{c o v}$, which is a sp-band Hubbard-like model[5]. The operators $H_{Q-P}$ and $H_{P-P}$ take into account the charge-dipole and dipole-dipole interactions[5,6,10].

By calculating size dependence of the ground state energy of $H$ by using an extended slave-boson method[5] we have determined the character of the bonding in $\mathrm{Hg}_{n}$ clusters. Small $\mathrm{Hg}_{n}$ clusters with $n \leq 10-20$, result $v d W$ bound: interatomic charge fluctuations are suppressed and the valence electrons are localized. As the cluster size increases, the Coulomb energy involved in charge fluctuations decreases due to the screening, which results mainly from the polarization of the surrounding atoms through charge-dipole interactions $H_{Q-P}$. At a critical cluster size $n_{c}\left(10 \leqq n_{c} \lessgtr 20\right)$ a transition from $v d w$ to covalent bonding occurs. The valence 


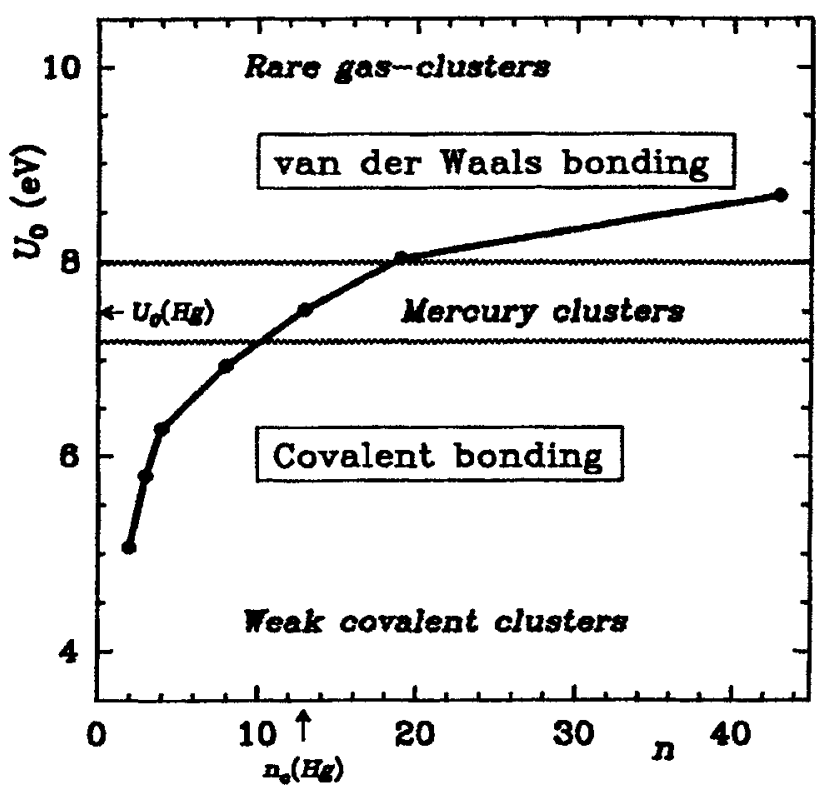

Figure 1: Phase diagram for $v d W$ and covalent bonding as a function of the intratomic Coulomb repulsion $U_{0}$ and $n$. Estimating $U_{0}\left(\mathrm{H}_{\mathrm{g}}\right)=7.5 \mathrm{eV}$, one obtains $n_{c}\left(\mathrm{Hg}_{\mathrm{g}}\right) \simeq 13$ atoms.

electrons delocalize and interatomic hopping is the dominant bonding mechanism.

In Fig. 1 we show a phase diagram for the bond character as a function of the cluster size and the intraatomic Coulomb energy $U_{0}=2 U_{s p}-U_{s s}$. Notice that the existence of a transition as a function of cluster size is restricted to a certain range of values for $U_{0}$. For instance, if $U_{0}<5 \mathrm{eV}$, the system would be covalent already for $n=2$, as it seems to be the case for $\mathrm{Be}_{n}$ clusters[7]. For $U_{0}>9 \mathrm{eV} \mathrm{vdW}$ behaviour results for all cluster sizes, as we obtain for rare-gas clusters. From the point of view of our theory, rare-gas clusters can be seen us an special case of closed-shell-atom clusters which retain the vdW character even for the bulk. It is one of the goals of our theory[5] to give a unified picture of the nature of the bonding in clusters of the various divalent metals and rare gases.

As already discussed above the ionization potential $I_{p}(n)$ is a magnitude which involves the electronic properties of both the neutral and the ionized clusters:

$J_{p}(n)=E^{+}(n)-E^{0}(n)$,

where $E^{0}(n)$ and $E^{+}(n)$ are the total binding energies of the neutral and the corresponding charged clusters. $I_{p}$ can be expressed in terms of binding energies as

$I_{p}(n)=I_{p}(1)-\left|D_{n}^{+}\right|+\left|D_{n}^{0}\right|$.

Here, $I_{p}(1)$ is the atomic ionization potential, $D_{n}^{0}$ the cohesive (dissociation) energy of the neutral cluster with respect to $n$ isolated nentral atoms, and $D_{n}^{+}$the cohesive energy of the ionized cluster with respect to an isolated ion and $(n-1)$ isolated neutral atoms.

Previous calculations of the $I_{p}(n)$ of rare-gas clusters are based on a model which considers the ionized rare-gas clnsters as consisting of a positively charged subcluster of $m$ atoms
( $m=2,3,4)$ surrounded by $(n-m)$ neutral atoms $[11,12]$. The hole created upon ionization tends to delocalize in order to decrease its kinetic energy. Notice that, in contrast to what occurs in neutral clusters, the delocalization of a single hole does not increase the Coulomb energy. Consequently, the gain in delocalization energy is large. However, after the hole is delocalized over $m$ atoms $(m<n)$, it could become energetically less favourable to delocalize the hole also on one more atom (i.e., on the $(m+1)$-atom) than to polarize this neutral atom around an ionized $m$-atom subcluster. This competition between hole delocalization and polarization energy is also contained in the Hamiltonian $H$ of Eq. (1), involving primarily the operators $H_{\text {cov }}$ and $H_{Q-P}$. Assuming that the hole is localized within a subcluster of $m$ atoms, the physics of cohesion can be described as follows. The positive charge of the ionized subcluster induces dipole moments in the $n-m$ neutral atoms, which remain vdW bound. The magnitnde and direction of each dipole are not only determined by the charge distribution of the ionized core but also by the interaction with the other dipoles through $H_{P-P}$. At the same time, the field produced by these dipole moments affects the dynamics of the hole, causing a shift of the energy levels for the hole on the different atoms of the ionized subcluster. This leads to a redistribution of the positive charge, which modifies again the induced dipoles and so on, until a balance between the kinetic energy of the hole, the charge-dipole attraction and the dipole-dipole interactions is reached.

Within the ionized subcluster, the hole is of dominant $s$ character and its kinetic energy is determined mainly by $8 s$ hopping[13], since the delocalization of a single hole does not involve Coulomb repulsion, and dipole-dipole energy is much smaller than the kinetic energy of the hole. Taking this into account, we can approximate the Hamiltonian of Eq. (1) for a singly ionized clusters[13] by

$$
\begin{aligned}
& \tilde{H}=(m-1)\left(2 \varepsilon_{s}^{0}+U_{s s}\right)-\sum_{t \in\{m\}}\left(\tilde{\varepsilon}_{s}+\sum_{j} \frac{e^{2} \alpha}{2 r_{l j}^{4}}\right) n_{l s \sigma}^{h}
\end{aligned}
$$

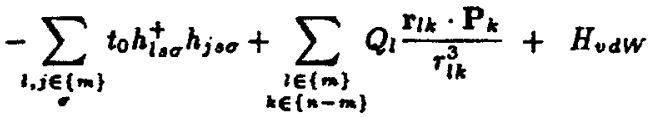

$$
\begin{aligned}
& +\frac{1}{2} \sum_{\substack{k, k^{\prime} \neq k \\
k, k^{\prime} \in(n-m\}}}\left[\frac{\mathbf{P}_{k} \cdot \mathbf{P}_{k^{\prime}}}{r_{k k^{\prime}}^{3}}-3 \frac{\left(\mathbf{r}_{k k^{\prime}} \cdot \mathbf{P}_{k}\right)\left(\mathbf{r}_{k k^{\prime}} \cdot \mathbf{P}_{k^{\prime}}\right)}{r_{k k^{\prime}}^{3}}\right] \text {, }
\end{aligned}
$$

where $h_{\text {log }}^{+}, h_{\text {lso }}$ and $n_{\text {log }}^{h}$ are the hole creation, annihilation and occupation operators, respectively. The ionized and neutral subclusters are represented by $\{m\}$ and $\{n-m\}$ respectively. Within $\{m\}$, charge-dipole interactions are taken into account only up to second order perturbation theory and thus result in a shift $\sum_{j} e^{2} \alpha / 2 r_{l j}^{4}$ of the on-site energies $\tilde{\varepsilon}_{4}$ at site $l$ with $j=$ nearest neighbours of $l$. $\tilde{\varepsilon}_{0}=\varepsilon_{0}^{0}+\Delta \varepsilon$, where $\varepsilon_{0}^{0}$ corresponds to the atomic level in the neutral atom, and $\Delta \varepsilon$ takes into account the shift produced in the energy levels by the absence of one electron. $t_{0}$ is the effective ss-hopping for the hole. The nentral subcluster is assumed to be purely $\mathrm{vdW}$ bound, i.e., we neglect the hopping elements between the neutral atoms and between the neutral and ionized subclusters[5].

We can rewrite $\tilde{H}$ in terms of the expectation values of the charge and dipole operators by expressing the terms con- 


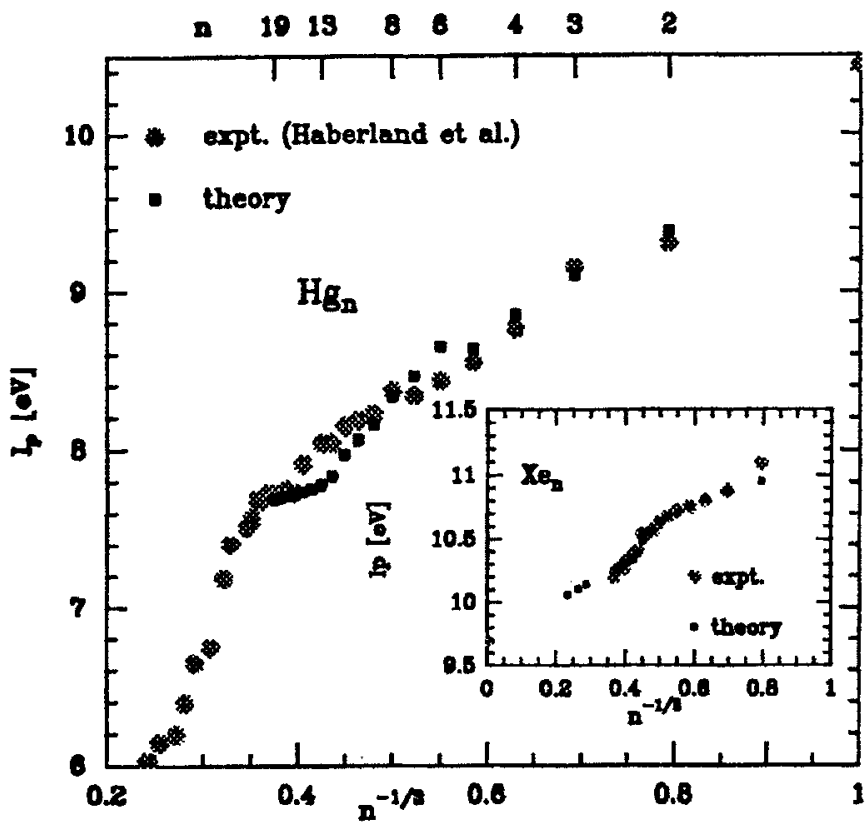

Figure 2: Calculated $I_{p}\left(\mathrm{Hg}_{n}\right.$ with $m=3$ and $t_{0}=0.7 \mathrm{eV}$, in comparison with electron-impact ionization results. Similar experimental results were obtained by photoionization (Ref. 1). Inset: calculated $I_{p}\left(\mathrm{Xe}_{n}\right.$ for $m=4$ and $t_{0}=0.15 \mathrm{eV}$ in comparison with experiment (Ref. 14).

taining four fermionic operators as $P_{k e} P_{k^{\prime} \eta}=P_{k e}\left\langle P_{k^{\prime} \eta}\right\rangle+$ $P_{k^{\prime} \eta}\left(P_{k e}\right)-\left\langle P_{k \varepsilon}\right\rangle\left\langle P_{k^{\prime} \eta}\right\rangle+\delta P_{k \varepsilon} \delta P_{k^{\prime} \eta}$, and $Q_{l} P_{k \varepsilon}=Q_{l}\left\langle P_{k \varepsilon}\right\rangle+$ $P_{k \in}\left\langle Q_{l}\right\rangle-\left\langle Q_{1}\right\rangle\left\langle P_{k e}\right\rangle+\delta Q_{l} \delta P_{k e}$. If one assumes that the vdW binding energy in the neutral and ionized clusters is roughly the same, the terms involving dipole-dipole fluctuations $\delta P_{k e} \delta P_{k^{\prime} \eta}$ together with $H_{v d W}$ cancel out in the calculation of the $I p(n)$ (see Eq. (3). Therefore, one obtains $I p(n)$ from the ground state of $H$ by calculating the chargedensity and dipole-density distributions self-consistently[10]. We assume vertical ionization. For the neutral clusters we use compact structures $[5,6]$, and for some cluster sizes we average the value of $I_{p}$ over different structures having the same total namber of bonds. We have calculated $I_{p}(n)$ of $\mathrm{Hg}_{n}$ clusters with $n \leq 19$ and for $X e_{n}$ clusters with $n \leq 300$. For the positively charged $m$-atom ionized cores we tested different sizes $(m=1,2,3,4)$ and shapes (e.g., linear and compact structures). We used $t_{0}(H g)=0.7 \mathrm{eV}$ and $t_{0}(X e)=0.15 \mathrm{eV}$, which yield the proper bulk band-width. The shift $\Delta \varepsilon$ (see Eq. (4), was determined by fitting to the experimental ionization potential of the trimer, in the case of $\mathrm{Hg}_{\mathrm{n}}$ clusters, to the average between $l_{p}(3)$ of Refs. 1 and 2 . Results for $I_{p}\left(\mathrm{Hg}_{n}\right)$ and $I_{p}\left(\mathrm{Xe}_{n}\right)$ are shown in Fig. 2 together with the corresponding experimental values[2,3,14]. For $\mathrm{Xe}_{n}$ clusters (inset figure), good agreement is obtained with experiment if one assumes a compact tetramer ionized core $(m=4)$. Note, that a rapid decrease in the $I_{p}(n)$ between $n=9$ and $n=13$, is observed for $\mathrm{Xe}_{n}$ clusters, both in the experimental and theoretical results. In our calculations, the positive charge (hole) is distributed over the tetramer for $n \leq 9$. For $n \geq 9$ most of the positive charge lies on the central atom yielding an increase of the polarization energy. Thus, a more pronounced decrease occurs in the ionization potential between $n=9$ and $n=13$, which is caused by a sharp increase of
$D^{+}(n)$ (see Eq. (3). Since for $n=13$ the 1st-neighbour shell of the central atom is completed, further addition of atoms $(n \geq 13)$ does not contribute appreciably to the polarization energy, and therefore the slope of $I_{p}(n)$ changes again to a lower value. Our results for the charge distribution in the ionized $\mathrm{Xe}_{n}$ clusters differ from those of Kuntz et al.[12]. They obtained that the ionized core becomes larger (e.g., changes from $m=3$ to $m=4$ ) for increasing cluster size, and that its structure is always linear.

Our results for the $I_{p}(n)$ of small $\mathrm{Hg}_{n}$ clusters are in good agreement with the experimental results of Haberland et al.[3] (electron-impact ionization) and of Rademann et al.[2] (photoionization), if one assumes a triangular trimer core $(m=3)$. We also obtain for $I_{p}\left(\mathrm{Hg}_{n}\right)$ a polarization decrease, which begins at $n=7$ and continues up to $n=13$. Its magnitude is larger than for $\mathrm{Xe}_{n}$ clusters, due to the larger atomic polarizability of $\mathrm{Hg}_{\mathrm{g}}$. A similar decrease can be also observed in the electron-impact ionization results[3], but in this case it occurs between $n=13$ and $n=15$. Note, that if we assume a linear trimer or tetramer for the ionized core, the agreement with experimental results is less satisfactory.

The fact that our calculated $I_{p}(n)$ agrees well with experiment up to $n=19$, suggests that $\mathrm{Hg}_{n}^{+}$clusters behave for $n \leq 20$ like ionized vdW clusters. For $n \geq 20$ this physical picture breaks down for $\mathrm{Bg}_{\mathrm{g}_{n}}$ as the electrons delocalize to form covalent bonds. Then, bond polarization and $s$-band broadening become important. A more rapid decrease of $I_{p}(n)$ occurs in the covalent region $(20 \leqslant n \leqslant 80)$, as shown in previous calculations [4,15]. Larger clusters $(n Z 80)$ show metallic behaviour, and a behaviour of the ionization potential according to the spherical droplet model[4]. This work was supported by the Deutsche Forschungsgemeinschaft through SFB 337 and 341.

\section{References}

1. C. Bréchignac, M. Broyer, Ph. Cahuzac, G. Delacretaz, P. Labastie, J. P. Wolf and L. Wöste, Chem. Phys. Lett. 120, 559 (1985), and Phys. Rev. Lett. 60, 275 (1988).

2. K. Rademann, B. Kaiser, U. Even and F. Hensel, Phys. Rev, Lett. 59, 2319 (1987).

3. H. Haberland, H. Kornemeier, H. Langosch, M. Oschwald and G. Tanner, J. Chem. Soc. Faraday Trans. 86, 2473 (1990).

4. G. M. Pastor, P. Stampli and K. H. Bennemann, Europhys. Lett. 7, 419 (1988) ; Phys. Scripta 38, 623 (1988).

5. M. E. Garcia, G. M. Pastor, and K. H. Bennemann, Phys. Rev. Lett. 67, 1142 (1991).

6. M.E. Garcia, PhD Thesis, Freie Universiät Berlin (1992).

7. R. Kawai and J. H. Weare, Phys. Rev. Lett. 65, 80 (1990).

8. S. N. Khanns, F. Reuse, and J. Buttet, Phys. Rev. Lett. 61, 535 (1988); C. Baladrón, M. E. Garcia, P. Stampli, and K. H. Bennemann, Z. Phys. D 19, 215 (1991).

9. F. London, Z. Phys, 63, 245 (1930).

10. M.E. Garcia, G.M. Pastor and K.H. Bennemann, submitted to Phys. Rev. B.

11. H. Haberland, Surf. Sci. 156, 305 (1985).

12. M. Amarouche, G. Durand, and J. P. Malrieu, J. Chem. Phys. 88, 1010 (1988); P. J. Kuntz and J. Valldorf, Z. Phys. D 8, 195 (1988); H. U. Böhmer et al., Z. Phys. D 11, 239 (1989).

13. The same calculations can be applied to a rare-gas-like cluster by interchanging the $s$ and $p$ orbitals in the equations (1)-(4). 14. G. Ganteför, G. Bröker, E. Holub-Krappe, and A. Ding, J. Chem. Phys. 91,7972 (1989).

15. A.A. Aligia et al, to be published. 